\title{
PENSAMIENTO MATEMÁTICO CREATIVO EN AULAS DE ENSEÑANZA PRIMARIA: ENTORNOS DIDÁCTICOS QUE POSIBILITAN SU DESARROLLO ${ }^{1}$
}

\author{
Paulina Araya², \\ Valentina Giaconi ${ }^{3}$, \\ María Victoria Martínez ${ }^{4}$
}

\begin{abstract}
RESUMEN
La creatividad matemática se relaciona con la capacidad de crear ideas, soluciones o preguntas que resultan novedosas desde la perspectiva de quien las genera. El desarrollo de esta habilidad es relevante en matemática a nivel profesional y escolar. Este artículo muestra los resultados de una investigación cuyo propósito fue determinar la influencia de los entornos didácticos en la creatividad matemática de los estudiantes. Para ello se evaluó la creatividad matemática de 576 estudiantes de $5^{\circ}$ básico pertenecientes a 21 cursos y 17 escuelas de Santiago de Chile y se emplearon modelos multinivel para indagar el efecto de distintos entornos didácticos. Los resultados muestran que el efecto del aula explica un $16 \%$ de la varianza total en la creatividad matemática de los estudiantes participantes. Una vez incorporados las variables de control "Nivel socioeconómico", "Género" y los resultados de "Simce", se observó que aquellos estudiantes que estuvieron en un entorno didáctico con una enseñanza caracterizada por involucrarlos de forma activa en la construcción de ideas y cuyos profesores mostraron una mayor capacidad para variar la dificultad de los problemas matemáticos, obtuvieron puntajes de creatividad matemática significativamente más altos. Estos hallazgos relevan la importancia del trabajo en el aula para el desarrollo del pensamiento matemático creativo.
\end{abstract}

Conceptos clave: aula matemática, creatividad, educación matemática, entornos didácticos, matemática.

\section{CREATIVE MATHEMATICAL THINKING IN PRIMARY EDUCATION CLASSROOMS: DIDACTIC ENVIRONMENTS THAT FAVOR ITS DEVELOPMENT}

\section{ABSTRACT}

Mathematical creativity is related to the ability to create ideas, solutions or questions that are novel, from the perspective of who generates them. The development of this skill is relevant in mathematics at both the professional and grade school level. This article shows the results of a study which sought to determine the influence of didactic environments on students' mathematical creativity. To this end, we evaluated the mathematical creativity of 5765 th grade students, from

1 Proyecto apoyado financieramente por CNED/Convocatoria 2017.

2 Universidad Alberto Hurtado, Santiago, Chile. Contacto: pau.araya.e@gmail.com

3 Universidad de O’Higgins, Rancagua, Chile. Contacto: valentina.giaconi@uoh.cl

4 Universidad de O'Higgins, Rancagua, Chile: contacto: mariavictoria.martinez@uoh.cl 

ENTORNOS DIDÁCTICOS QUE POSIBILITAN SU DESARROLLO - P. Araya, V. Giaconi, M.V. Martínez

21 cohorts and 17 schools in Santiago de Chile, and used multilevel models to investigate the effect of different teaching environments. The results show that the classroom effect helps to explain $16 \%$ of the total variance in the mathematical creativity of the participating students. Once the control variables "Socioeconomic Level", "Gender" and "Simce" (standardized test results) were incorporated, it was observed that those students who were in a didactic environment with teaching characterized by actively involving them in the construction of ideas and whose teachers showed a greater capacity to vary the difficulty of the mathematical problems, obtained significantly higher scores of mathematical creativity. These findings reveal the importance of classroom experiences for the development of creative mathematical thinking.

Key concepts: creativity, didactic environments, mathematics, mathematical classroom, mathematics education.

\section{Introducción}

En los últimos 30 años ha surgido un interés de la comunidad de investigadores en educación por comprender y conceptualizar el papel de la creatividad en la matemática y su aprendizaje (Leikin \& Pitta Pantazzi, 2013; Singer, Sheffield \& Leikin, 2017). Al mismo tiempo, la capacidad creativa como habilidad a ser desarrollada en ambientes escolares ha sido foco de algunas políticas públicas, por ejemplo: uno de los objetivos de la educación matemática declarado por la Unión Europea es promover el pensamiento matemático creativo en los estudiantes, tanto a nivel del aula como de los sistemas educativos en general (European Commission, EC, 2006). En Chile, las bases curriculares señalan que la matemática es una disciplina creativa y multifacética, e indican que la creatividad es una actitud que debe ser desarrollada a lo largo de su enseñanza (Ministerio de Educación de Chile, Mineduc, 2012).

En un mundo cambiante, de rápidos avances tecnológicos y científicos que modifican las vidas de las personas, la creatividad es necesaria tanto para adaptarse como para realizar nuevos avances y, en general, permite enfrentar los retos que aparecen en múltiples áreas de la vida y generar aportes a los diversos campos disciplinares. La creatividad matemática (en adelante CM) es un tipo específico de creatividad que resulta central en los avances tanto de las diferentes ramas de la matemática como de las áreas científicas que la emplean y que permiten sostener el progreso tecnológico y científico (Leikin, 2013). 
Distintos autores señalan la importancia de la creatividad en la actividad matemática a nivel profesional, ya que todas las producciones matemáticas son fruto de procesos creativos que culminaron en contribuciones nuevas, y donde no fue suficiente el correcto empleo de los algoritmos y procedimientos conocidos para llegar a soluciones acertadas (Dreyfus \& Eisenberg, 1996; Ginsburg, 1996; Mann, 2006; Sriraman, 2009).

Si bien, el trabajo matemático en la etapa escolar no tiene por objetivo generar contribuciones nuevas a este campo, es posible, a través de la educación matemática escolar, estimular el pensamiento creativo de los estudiantes, por ejemplo, mediante actividades como la invención y resolución de problemas (Kattou, Kontoyianni, Pitta-Pantazi \& Christou, 2013; Liljedahl, 2016; Mann, 2006; Sinclair, Freitas \& Ferrata, 2013; Voica \& Singer, 2013). Propiciar el desarrollo de la creatividad en el aula favorece el interés de los jóvenes, al apartarlos de una visión de la matemática como una disciplina reiterativa y mecanicista (Mann, 2006; Sheffield, 2017).

Las experiencias creativas en la construcción de un conocimiento matemático tienen implicancias en el plano afectivo de los estudiantes y su relación con la disciplina (Hannula et al., 2016; Liljedahl, 2013; Mann, Chamberlin \& Graefe, 2017). La investigación de Liljedahl (2013) mostró que jóvenes con rechazo manifiesto hacia la matemática mostraron un cambio afectivo favorable después de haber tenido experiencias creativas en la producción de ideas matemáticas. Este aspecto es relevante ya que la disposición afectiva hacia la matemática condiciona los procesamientos cognitivos de los sujetos y determina el éxito que un estudiante va a experimentar con la disciplina (Hannula, 2014; Linnenbrink \& Pintrich, 2004).

La CM es una característica plástica y no un rasgo predeterminado (Sheffield, 2017) y, además, puede ser fomentada o inhibida mediante factores ambientales, es decir, por medio de experiencias como las que tienen lugar en la familia y en la escuela (Leikin, 2013; Leikin \& Pitta-Pantazi, 2013; Sheffield, 2013). Dentro de esos factores ambientales los que tienen que ver con la experiencia escolar son el objeto de este estudio. Diversas investigaciones 

ENTORNOS DIDÁCTICOS QUE POSIBILITAN SU DESARROLLO - P. Araya, V. Giaconi, M.V. Martínez

muestran el efecto que tiene el aula en el desarrollo de la CM de los estudiantes (Kanhai \& Singh, 2016; Kwon, Park \& Park, 2006; Sarrazy \& Novotná, 2013; Tabach \& Friedlanyer, 2013). Estos estudios han empleado análisis cuantitativos identificando que la variabilidad en las evaluaciones de creatividad es explicada en parte por el agrupamiento a nivel curso; en otras palabras, las características particulares de la enseñanza inciden de algún modo en el desarrollo de la CM de sus estudiantes. Sin embargo, son escasas las investigaciones que abordan el tipo de enseñanza matemática que resulta favorable para fomentar el potencial creativo de sus estudiantes.

Por otro lado, investigaciones en didáctica de la matemática sugieren aspectos de la enseñanza que podrían favorecer el desarrollo del aprendizaje y la creatividad (Brousseau, 2007; Sarrazy \& Novotná, 2013). Por ejemplo, el concepto de entornos didácticos (Sarrazy \& Novotná, 2013) describe el aula matemática centrándose en el estudio de algunos aspectos de la clase, como el tipo de tareas que se presentan a los estudiantes, el tipo de retroalimentación que ofrecen los profesores, la forma en que se aborda la incorporación de contenidos nuevos, la organización social del aula, entre otros. Sin embargo, son escasas las investigaciones que se centran en la relación entre estos elementos de los entornos didácticos y la creatividad matemática de los estudiantes.

En esta línea, el presente estudio se pregunta de qué manera los entornos didácticos donde se desenvuelven los estudiantes influyen en su capacidad de desplegar pensamiento matemático creativo. Siguiendo la literatura en el campo, esta investigación, por un lado, evalúa la CM de los estudiantes y, por otro, caracteriza el trabajo en el aula a través del lente teórico de entornos didácticos, permitiendo hallar relaciones entre ambos constructos e identificando aquellos tipos de entornos didácticos que favorecen el pensamiento matemático creativo en sus estudiantes. 


\section{Marco conceptual}

\subsection{Creatividad matemática}

Distintos autores señalan que el concepto de CM, al igual que el concepto de creatividad, no tiene una definición ampliamente aceptada por la literatura (Barquero, Richter, Barajas y Font, 2014; Singer, Sheffield \& Leikin, 2017). Pese a la falta de una definición precisa, todas las aproximaciones teóricas reconocen la creatividad como una habilidad humana que permite la creación de ideas nuevas que modifican o incrementan lo existente (Csizenmihalyi, 2000; Torrance, 1974). La CM es una creatividad específica que tiene en cuenta la naturaleza lógica del campo (Leikin, 2013; Piirto, 1999).

La CM es una de las características del pensamiento matemático avanzado, que se refleja en la capacidad de formular objetivos matemáticos y encontrar relaciones inherentes entre ellos (Ervynck, 1991; Lithner, 2008). Naturalmente, la capacidad creativa que puede experimentarse en contextos escolares difiere de la de los matemáticos profesionales (Leikin, 2013; Lev-Zamir \& Leikin, 2013). Liljedahl y Sriraman (2006) distinguen entre la CM a nivel profesional, que tiene lugar dentro de la comunidad académica y que genera aportes en alguna de las distintas ramas de la matemática (o big-creativity), y la CM a nivel de enseñanza y aprendizaje en contextos escolares, donde los aportes consisten en ideas que resultan novedosas desde la perspectiva de los estudiantes (little-creativity). Este segundo nivel, que es el de interés en el presente trabajo, es definido como "el proceso que da como resultado soluciones inusuales (nuevas) y/o perspicaces a un problema dado, y/o la formulación de nuevas preguntas y/o posibilidades que permiten considerar un viejo problema desde un nuevo ángulo" (Liljedah \& Sriraman, 2006, p. 195).

En efecto, los estudiantes en etapa escolar pueden ofrecer ideas que son novedosas respecto de la matemática que han aprendido y de los problemas que han resuelto. Sternberg (2004) sostiene que los procesos mentales que inciden en la big-creativity y en la littlecreativity son psicológicamente equivalentes, lo que distingue uno

Traducción propia. 

ENTORNOS DIDÁCTICOS QUE POSIBILITAN SU DESARROLLO - P. Araya, V. Giaconi, M.V. Martínez

del otro es la interacción entre el resultado del proceso creativo y el contexto. Ciertamente, los estudiantes pueden experimentar procesos creativos en matemática aun cuando no lleguen a resultados que logren extender su campo: el hecho de que logren modificar y extender el campo de lo conocido por ellos mismos representa un proceso cognitivo altamente complejo y valioso.

Por otra parte, distintas investigaciones han tenido por objetivo desarrollar instrumentos que permitan evaluar la CM a nivel escolar. Estas parten de la premisa que resolver problemas matemáticos de distintas maneras está estrechamente relacionado con la capacidad creativa, y sugieren evaluarla mediante problemas de solución múltiple (Ervynck, 1991; Kattou et al., 2013; Kwon, Park \& Park, 2006; Leikin, 2013; Lev-Zamir \& Leikin, 2013).

Un problema de solución múltiple corresponde a un problema matemático que tiene muchas o infinitas respuestas correctas y que permite distintas estrategias o perspectivas de resolución (Leikin, 2013; Nohda, 2000; Pehkonen, 1997). El conjunto de las soluciones producidas por cada sujeto puede ser evaluado sobre la base de las categorizaciones que propone Torrance (1974):

- Fluidez, o cantidad de ideas matemáticas o soluciones que un sujeto produce en respuesta a una situación.

- Flexibilidad, o cantidad de enfoques o estrategias distintas que el sujeto genera en respuesta a una situación.

- Originalidad, entendida como la rareza de la estrategia usada, se otorga mayor puntaje a las estrategias menos empleadas.

A partir de estas categorías es factible generar rúbricas que evalúen las soluciones de los estudiantes en cada dimensión, permitiendo otorgar puntajes al desempeño creativo.

\subsection{Entornos didácticos}

Con la finalidad de analizar el trabajo en el aula de matemática se empleará el enfoque teórico planteado por Sarrazy y Novotná (2013), quienes han definido distintos criterios para el análisis didáctico del aula matemática bajo el concepto de entornos didácticos. Los autores 
emplean conceptos provenientes de la tradición de didáctica francesa (Brousseau, 2007) y, a partir de ellos, establecen las dimensiones que capturan la riqueza didáctica de una clase. Específicamente, el concepto de contrato didáctico permite a los autores relacionar el aula con el pensamiento matemático creativo y corresponde a las normas implícitas y explícitas que regulan la relación de los estudiantes con el saber matemático. Como producto de la fuerza de estas normas, estos no se vinculan únicamente con el saber matemático, sino con lo que creen que sus profesores esperan de ellos (Brousseau, 2007; Brousseau, Sarrazy \& Novotná, 2014). En consecuencia, los estudiantes intentan cumplir las expectativas de los maestros, y no necesariamente intentan generar ideas novedosas, si no que analizan e identifican lo que el profesor aprobará respecto de sus producciones matemáticas.

El contrato didáctico que emerge entre un profesor y su grupo de estudiantes se conforma a partir de la repetición de las experiencias que tienen lugar en el aula en relación con el conocimiento, y se instala paulatinamente. Según Brousseau (2007) el contrato didáctico que se ha asentado de manera progresiva entre un profesor y su grupo de estudiantes, pese a compartir ciertos atributos generales, posee características que son propias de un grupo en particular y, por lo tanto, debe estudiarse de manera situada en cada aula. Basándose en esta premisa Novotná y Sarrazy $(2010 ; 2011)$ desarrollan un modelo de análisis del aula que caracteriza los distintos contratos didácticos a partir de la descripción de diversos aspectos observados en la sala de clases, aunados bajo el concepto de entornos didácticos.

Los entornos didácticos corresponden a las prácticas docentes descritas a partir de distintas dimensiones observadas en el aula que configuran un perfil de acciones del profesor (Novotná \& Sarrazy, 2010; 2011). Tales dimensiones pueden ser exploradas a partir de lo que el maestro realiza en la clase de matemática y de su capacidad para variar la dificultad en los problemas matemáticos que emplea.

Según Sarrazy y Novotná (2005; 2013) cada entorno puede describirse a partir de seis dimensiones, cinco de ellas asociadas a aspectos didácticos y organizacionales del aula, las que son observadas 
directamente en la sala de clases. La sexta dimensión describe la capacidad del profesor de variar la dificultad de los problemas matemáticos, por lo que se obtiene del análisis de problemas creados por el profesor. Estas dimensiones son:

- Complejidad de las tareas: las tareas empleadas por el profesor durante la clase condicionan las acciones de los estudiantes y enmarcan la actividad matemática en el aula. El nivel de complejidad de la tarea determina en gran medida la demanda cognitiva que el estudiante experimentará en la clase. La dificultad de una tarea, si bien depende del nivel de conocimientos y experiencias previas de un alumno, a modo general puede clasificarse como: tareas triviales, que requieren el recuerdo de nociones que los estudiantes ya conocen y que es posible observarlas en preguntas que el profesor realiza y que toda la clase responde a coro sin dificultad; tareas de aplicación directa, que requieren de la ejecución de un procedimiento conocido por el estudiante; preguntas contextualizadas, que requieren que el estudiante establezca una relación entre tópicos matemáticos y situaciones elaboradas en contextos extra matemáticos; y tareas de descubrimiento o problema, que corresponden a aquellas preguntas cuya solución o método de solución no es conocido a priori por el estudiante y que requiere de un análisis más detenido (Sarrazy \& Novotná, 2010; Smith \& Stein, 1998).

- Institucionalización del saber: la teoría de situaciones didácticas (Brousseau, 2007) denomina institucionalización al momento donde el profesor conecta las ideas matemáticas que han logrado construir sus estudiantes vinculándolas con la matemática oficial. En esta dimensión se describe la manera en que se abordan los conocimientos matemáticos de la clase, observando si los procedimientos y nociones matemáticas son construidos entre el profesor y los estudiantes, o si el profesor las presenta de manera expositiva. De este modo, las nociones matemáticas pueden ser presentadas de forma directa por el profesor, sin ninguna intervención de los alumnos; o bien después de preguntas triviales que requieren que los estudiantes reconstruyan recuerdos de clases anteriores; también se pueden integrar ideas de los estudiantes mediante una pregunta que promueva el pensamiento 
y, en casos más sofisticados, los conocimientos pueden ser el producto del trabajo de los estudiantes en un problema, cuya solución son los contenidos que deben de aprender, en la línea de la teoría de situaciones didácticas.

- Modos de validación de las producciones: una producción matemática es una idea o una pregunta elaborada por un estudiante durante la clase y que guarda relación con la matemática. Esta dimensión aborda la manera en que el profesor reacciona frente a las producciones de sus alumnos durante la clase. Estas reacciones pueden ir desde formas que requieren más o menos actividad de parte de los estudiantes. El profesor puede ignorar la producción del estudiante; puede validarla directamente diciendo que una idea es correcta o incorrecta; puede explicar una respuesta explicitando las razones de por qué una idea es correcta o incorrecta; puede pedir que sus estudiantes justifiquen por qué una respuesta es correcta o incorrecta y puede devolver una pregunta realizada por un estudiante para que esta sea reflexionada por la clase. Estas formas van en coherencia con lo que Brousseau (2007) define como momento a-didáctico, donde el rol del maestro consiste en devolver a los estudiantes la responsabilidad de generar por sí mismos las nociones matemáticas. Si bien, esta dimensión se relaciona con la anterior, puesto que ambas observan la interacción entre profesor y estudiantes y su rol en la construcción del conocimiento, esta atiende a interacciones por lo general más breves que suelen ocurrir durante toda la clase.

- Posibilidad de comunicación: en una sala en que la comunicación se establece mayoritariamente entre profesor y alumno, los estudiantes suelen adoptar un rol más pasivo y receptivo, mientras que en los cursos donde se intenciona el diálogo entre estudiantes, quienes están en una misma jerarquía con respecto al conocimiento, estos adoptan por lo general roles más activos en la discusión y en la construcción de ideas. Esta dimensión captura las posibilidades que tienen los estudiantes de discutir ideas matemáticas con los distintos actores de la sala de clases.

- Disposición organizativa: atendiendo a la organización del aula, esta dimensión observa el modo en que el maestro dispone la 

ENTORNOS DIDÁCTICOS QUE POSIBILITAN SU DESARROLLO - P. Araya, V. Giaconi, M.V. Martínez

sala de clases y cómo acomoda los puestos de los estudiantes, pudiendo estar sentados en grupos, en pares o de manera individual.

La sexta dimensión guarda relación con la capacidad que tienen los maestros de primaria para generar problemas matemáticos que varían en su dificultad. Sarrazy (2002) muestra la importancia de esta capacidad explicando que los maestros que emplean problemas o tareas poco variadas llevan a una hiperadaptación de sus alumnos a las situaciones propuestas. Para adaptarse a las demandas habituales del maestro, los alumnos desarrollan estrategias de afrontamiento sobre la base de criterios usualmente utilizados. Los estudiantes pueden detectar fácilmente indicadores que les permitan adaptar sus decisiones y su comportamiento a las solicitudes didácticas de sus maestros. En ese caso, los estudiantes pueden aplicar un comportamiento adecuado sin entender exactamente el sentido de la lección o del problema que se les asignó. Contrariamente, en caso de fuerte variabilidad, el compromiso de los estudiantes en la situación es mucho más probable. El autor recopila investigaciones que apuntan a identificar la dificultad de los problemas matemáticos escritos, acotándolos al ámbito aritmético, y distingue 14 variables agrupadas en variables numéricas, retóricas y semántico-conceptuales.

- Variables numéricas: relacionadas con los aspectos numéricos, observa si el problema requiere de la ejecución de algoritmos o no, y si en su redacción tiene números que deban ignorarse o distractores para resolver el problema correctamente.

- Variables retóricas: relacionadas con la forma en que se organiza la presentación del problema, es decir, el tipo de vocabulario empleado, si contiene verbos que indiquen la operación matemática a realizar (índice semántico), la familiaridad del tema, si su formulación es concisa o abunda en detalles narrativos y si el orden en que se presentan los hechos en el texto coincide con el orden temporal narrado.

- Variables semántico-conceptuales: relacionadas con aspectos estrictamente lógico-matemáticos, por ejemplo, el tipo de estructura aditiva (Vergnaud, 1983), el vínculo entre el índice semántico y el operador, el vínculo entre el orden en el que se 
presentan los números en el problema y el orden en que deben escribirse los datos para realizar el cálculo correcto.

La examinación de cada una de estas variables en problemas redactados por los profesores permite identificar la capacidad que tiene el maestro para plantear a sus alumnos problemas que varíen en su dificultad.

\section{Objetivos de la investigación}

Los objetivos de esta investigación han sido organizados a partir de un objetivo general y cuatro objetivos específicos.

\section{Objetivo general}

Caracterizar la manera en que los entornos didácticos donde se desenvuelven los estudiantes influyen en su capacidad de desplegar pensamiento matemático creativo.

\section{Objetivos específicos}

- Evaluar la creatividad matemática de estudiantes al enfrentar la resolución de problemas de solución múltiple.

- Caracterizar los entornos didácticos en los que se desenvuelven los estudiantes cuando trabajan en el aula de matemática.

- Relacionar la creatividad matemática de los estudiantes y los entornos didácticos en los que se desenvuelven.

- Caracterizar los entornos didácticos que favorecen o inhiben la creatividad matemática de los estudiantes.

\section{Metodología}

El enfoque metodológico empleado en este estudio consistió en un análisis multinivel, conocido también como análisis lineal jerarquizado (Bickel, 2007; Murillo, 2008) con dos niveles: los estudiantes (que corresponden al nivel 1) y el aula (que corresponde al nivel 2). Estos modelos permiten estudiar el efecto de variables independientes en una variable dependiente, en donde las primeras pueden corresponder al nivel 1 (por ejemplo, sexo) o al nivel 2 (características del aula). 
Según Murillo (2008), los modelos multinivel son la metodología de análisis más adecuada para trabajar con datos jerarquizados o anidados (por ejemplo, estudiantes en aulas, aulas en escuelas), esto los convierte en una estrategia adecuada para la investigación educativa de carácter cuantitativo, en los casos donde se requiere conocer cómo se relacionan las características del curso asociadas al profesor, o a una escuela con una variable dependiente. Esto posibilita realizar análisis tales como estimar cuánta varianza de los datos explica cada nivel de análisis en la variable dependiente (efecto del aula o la escuela) o la influencia de variables independientes de distintos niveles.

Para este estudio, la CM de los estudiantes será explicada a partir de las características de los entornos didácticos, incorporando variables de control como el género, el nivel socioeconómico (NSE) de la escuela y el promedio del colegio obtenido en una prueba estandarizada nacional de matemática aplicada el año anterior al estudio (en adelante Simce $4^{\circ}$ año básico 2017). La variable dependiente, la CM de los estudiantes, se ve influenciada por el aula según la literatura, por lo que es necesario considerar su variación a nivel del estudiante y del aula. De este modo, el modelo multinivel representa un modo de análisis adecuado para el objetivo de esta investigación.

\subsection{Participantes}

Los cursos participantes en este estudio fueron seleccionados a partir de un muestreo no probabilístico intencional. La muestra tuvo por objetivo incluir escuelas con distintos perfiles y pertenecientes a distintos niveles socioeconómicos. Dado que el propósito de este estudio se enfocó en el análisis de la creatividad, se incluyeron en la muestra algunas escuelas con un perfil "alternativo" como son las escuelas artísticas, Montessori o Freinet, que en sus proyectos educativos manifiestan abiertamente el interés por desarrollar la creatividad en sus estudiantes.

Se eligió el nivel $5^{\circ}$ año básico para realizar el estudio por dos razones: por un lado, los instrumentos diseñados para evaluar CM 
han sido elaborados mayoritariamente para niños de 10 a 12 años y, por otro, el nivel $5^{\circ}$ año básico cuenta con la evaluación Simce realizada en $4^{\circ}$ año básico del año anterior al estudio.

Se hizo un llamado masivo por medio de redes sociales a profesores que realizaran clases de matemática en $5^{\circ}$ año básico de escuelas tradicionales y alternativas, invitándolos a participar de esta investigación; su interés tendría como retribución un taller docente acerca del tema. La muestra se compuso con los profesores que respondieron a dicho llamado.

Participaron 21 cursos de nivel $5^{\circ}$ año básico correspondientes a 17 escuelas de la Región Metropolitana, Santiago, Chile. El número total de estudiantes fue de 576, tamaño de muestra similar a otros estudios que han empleado análisis multinivel para determinar efecto aula (Joët, Usher \& Breassoux, 2011). Los cursos participantes se distribuyeron según perfil de la escuela y NSE como se muestra en la Tabla 1.

Tabla 1

Distribución de los cursos participantes según nivel socioeconómico y perfil de la escuela

\begin{tabular}{llllll}
\hline \multicolumn{2}{l}{ Escuelas tradicionales } & & & \multicolumn{2}{l}{ Escuelas alternativas } \\
\hline NSE alto & $\begin{array}{l}\text { NSE medio } \\
\text { alto }\end{array}$ & NSE medio & $\begin{array}{l}\text { NSE medio } \\
\text { bajo }\end{array}$ & NSE alto & NSE medio \\
\hline 7 & 2 & 8 & 1 & 2 & 1 \\
\hline
\end{tabular}

Fuente: Elaboración propia.

\subsection{Instrumentos}

\subsubsection{Test de creatividad matemática}

Para evaluar CM se diseñó y adaptó un instrumento a partir de una selección de preguntas presentes en los test validados por Sighn (1987); Lee, Hwang y Seo (2003); Tabach y Fiedlander (2013), y Kwon, Park y Park (2006). Estos test emplean problemas de solución múltiple, los que permiten a los estudiantes producir un conjunto de soluciones para cada pregunta. 

ENTORNOS DIDÁCTICOS QUE POSIBILITAN SU DESARROLLO - P. Araya, V. Giaconi, M.V. Martínez

Las preguntas fueron elegidas según su dificultad y pertinencia para el contexto local, y fueron piloteadas previamente para asegurar que los estudiantes comprendieran las instrucciones. La versión piloto del test fue aplicada a un curso de 42 estudiantes de $5^{\circ}$ año básico pertenecientes a una escuela que no correspondía a la muestra, eliminándose dos preguntas por su excesiva dificultad. La versión final del instrumento (ver Anexo 1) se construyó usando los ítems que mostraron un buen funcionamiento en el piloto. El instrumento fue aplicado por el equipo de investigación en los 21 cursos participantes siguiendo un protocolo de aplicación que aseguró la misma explicación de los ítems y el mismo tiempo de desarrollo (60 minutos) para todos los estudiantes.

Las respuestas de los estudiantes fueron analizadas mediante la codificación en las tres dimensiones de CM: fluidez, flexibilidad y originalidad, siguiendo las rúbricas de puntuación adaptadas de los test originales. Fluidez correspondió a un puntaje asignado por la cantidad de respuestas correctas diferentes. Flexibilidad se obtuvo a partir de la suma de las distintas estrategias empleadas por los estudiantes en sus soluciones. El puntaje asignado a la dimensión originalidad se estableció dando mayor puntaje a las estrategias empleadas por una menor cantidad de estudiantes. Las rúbricas de puntuación se detallan en el Anexo 2.

Los puntajes de las tres dimensiones fueron sumados para obtener el puntaje total de cada alumno. Los puntajes asignados a cada dimensión para cada uno de los tres ítems del instrumento generaron un total de nueve indicadores por alumno, se estimó el coeficiente alfa de Cronbach con estos nueve indicadores y resultó en 0,73 , lo que muestra una confiabilidad adecuada. Respecto de las correlaciones ítem-test, estas estuvieron en un rango entre 0,274 y 0,589 , lo que indica que todos los indicadores considerados discriminan apropiadamente.

\subsubsection{Modelo de análisis de entornos didácticos}

Se filmaron dos clases consecutivas de matemática de cada curso participante. Cada profesor realizó clases normales según su planificación y el contenido a tratar no fue designado por el equipo 
de investigación. Estas clases fueron posteriormente analizadas según una pauta de observación de clases basada en el modelo de análisis de clases creado por Sarrazy \& Novotná (2013). La pauta examinó cinco dimensiones en segmentos de 20 minutos y las puntuó entre 0 y 1. Finalmente, se promediaron los puntajes de los segmentos para obtener el puntaje final de cada una de las cinco dimensiones para cada profesor. Las dimensiones observadas son las siguientes:

- Dificultad de las tareas: observa si el profesor emplea tareas triviales, de aplicación directa, contextualizadas o problemas no triviales. En cada segmento, se asignó el puntaje asociado a la tarea o pregunta de mayor dificultad dada por el profesor.

- Institucionalización del saber: observa la manera en que el profesor enseña los conceptos y nociones matemáticas en relación con la participación más o menos activa de los estudiantes en la construcción de ese saber. En cada segmento un puntaje más cercano a 1 indica un mayor involucramiento de los estudiantes en el establecimiento de las ideas matemáticas.

- Modos de validación de las producciones de los estudiantes: se observó la reacción del profesor ante las producciones o preguntas de los estudiantes. En cada segmento un puntaje más cercano a 1 indica que el profesor reaccionó pidiendo una mayor participación de los estudiantes: por ejemplo, justificar una idea matemática o devolvió la pregunta a los estudiantes; mientras que un puntaje de 0 indica una mayor pasividad: por ejemplo, el profesor da las respuestas, el profesor ignora preguntas, etc.

- Posibilidades de comunicación: se observó con quiénes se comunicaron los estudiantes en la clase, con el compañero, con toda la clase, con el profesor. Un puntaje más cercano a 1 indica que los estudiantes pudieron comunicarse con más actores de la clase, y un puntaje más cercano 0 indica menor interacción social.

- Disposición organizativa: se observó el modo en que fueron dispuestos los estudiantes: en grupos, en pares, de a uno. Un puntaje más cercano a 1 indica que los estudiantes estuvieron sentados en grupos por más tiempo, y un puntaje más cercano a 0 indica que estuvieron sentados solos o una mayor parte del tiempo. 

ENTORNOS DIDÁCTICOS QUE POSIBILITAN SU DESARROLLO - P. Araya, V. Giaconi, M.V. Martínez

3.2.3. Variabilidad en la dificultad de las tareas creadas por el profesor Para evaluar esta sexta dimensión se solicitó a los profesores participantes crear tres problemas para sus estudiantes de $5^{\circ}$ año básico que se resolvieran usando adición, y tres problemas que se resolvieran usando sustracción. Estos fueron analizados a partir de las variables descritas por Sarrazy (2002), las que se indican en la Tabla 2.

Tabla 2

Descripción de variables sobre la variabilidad en la dificultad de las tareas

\begin{tabular}{|c|c|c|}
\hline Tipo de variable & \multicolumn{2}{|c|}{ Modalidades evaluadas } \\
\hline \multicolumn{3}{|l|}{ Variables numéricas } \\
\hline 1. Tipos de números utilizados & Requiere algoritmo & No requiere \\
\hline 2. Datos irrelevantes & Presencia & Ausencia \\
\hline \multicolumn{3}{|l|}{ Variables retóricas } \\
\hline 3. Índice semántico & Presencia & Ausencia \\
\hline 4. Contextos familiares & Presencia & Ausencia \\
\hline 5. Disparador semántico & Presencia & Ausencia \\
\hline $\begin{array}{l}\text { 6. Organización sintagmática y } \\
\text { organización temporal }\end{array}$ & Coincide & No coincide \\
\hline 7. Posición de la pregunta & Medio & Final \\
\hline 8. Vocabulario & Conocido & Desconocido \\
\hline 9. Formulación & Clásico & Narrativo \\
\hline \multicolumn{3}{|l|}{ Variables semántico-conceptuales } \\
\hline 10. Estructura aditiva & 1 & 4 \\
\hline 11. Naturaleza de lo desconocido & Inicial & Final \\
\hline $\begin{array}{l}\text { 12. Correspondencia orden } \\
\text { sintagmático y orden operativo }\end{array}$ & Coincidencia & No coincidencia \\
\hline $\begin{array}{l}\text { 13. Correspondencia } \\
\text { desencadenante y operador } \\
\text { matemático }\end{array}$ & Coincidencia & No coincidencia \\
\hline $\begin{array}{l}\text { 14. Correspondencia índice } \\
\text { semántico y el operador } \\
\text { matemático }\end{array}$ & Coincidencia & No coincidencia \\
\hline
\end{tabular}

Fuente: Elaboración propia.

Para calcular el puntaje de variabilidad se siguió el procedimiento utilizado en Sarrazy (2002) que consistió en contar, para cada una de las 14 variables, las variaciones observadas (Vo) sobre los tres problemas de adición en su conjunto, en relación con el número de variaciones posibles (Vp). Así, cada variable obtiene un índice $\mathrm{I}=\mathrm{Vo} / \mathrm{Vp}$. El mismo análisis se realizó para los problemas de 
sustracción. Se calculó el promedio aritmético de las variables numéricas, retóricas y semántico conceptuales. Finalmente, el índice quedó determinado por la fórmula:

$$
\mathrm{I}=\frac{\sum_{i=1}^{2} \frac{v o i}{v p i}}{2}+\frac{\sum_{i=3}^{9} \frac{v o i}{v p i}}{7}+\frac{\sum_{i=4}^{14} \frac{v o i}{v p i}}{5}
$$

\subsection{Análisis estadísticos}

En esta sección se describen en detalle los análisis estadísticos realizados en el estudio. Las variables consideradas en el análisis y el total de datos perdidos se describen en la Tabla 3. Respecto de los datos perdidos, se presentaron dos casos: un valor perdido para la variable D6, ya que una profesora no respondió la actividad de invención de problemas, y el promedio en la prueba Simce de una escuela alternativa no reconocida por el Mineduc. Para no perder la información del curso completo se optó por imputar estos valores con la media aritmética del resto de los casos.

Tabla 3

Descripción de las variables y del total de perdidos

\begin{tabular}{|c|c|c|c|c|c|}
\hline Variable & $\begin{array}{c}\text { Nombre } \\
\text { para los } \\
\text { análisis } \\
\text { estadísticos }\end{array}$ & Nivel & Tipo & Valores & $\begin{array}{c}\mathrm{N} \\
\text { Perdidos }\end{array}$ \\
\hline $\begin{array}{l}\text { Creatividad } \\
\text { matemática }\end{array}$ & y & Estudiante & Numérica & - & 0 \\
\hline Sexo & sexo & Estudiante & Categórica & $\begin{array}{c}\text { Hombre } \\
\text { Mujer }\end{array}$ & 0 \\
\hline Dificultad de las tareas & D1 & Clase & Numérica & - & 0 \\
\hline $\begin{array}{l}\text { Institucionalización } \\
\text { del saber }\end{array}$ & D2 & Clase & Numérica & - & 0 \\
\hline $\begin{array}{l}\text { Modos de validación } \\
\text { de las producciones de } \\
\text { los estudiantes }\end{array}$ & D3 & Clase & Numérica & - & 0 \\
\hline $\begin{array}{l}\text { Posibilidades de } \\
\text { comunicación }\end{array}$ & D4 & Clase & Numérica & - & 0 \\
\hline $\begin{array}{l}\text { Disposición } \\
\text { organizativa }\end{array}$ & D5 & Clase & Numérica & - & 0 \\
\hline $\begin{array}{l}\text { Indice de variabilidad } \\
\text { en la dificultad de las } \\
\text { tareas }\end{array}$ & D6 & Clase & Numérica & - & 1 \\
\hline
\end{tabular}


336 PENSAMIENTO MATEMÁTICO CREATIVO EN AULAS DE ENSEÑANZA PRIMARIA: ENTORNOS DIDÁCTICOS QUE POSIBILITAN SU DESARROLLO - P. Araya, V. Giaconi, M.V. Martínez

\begin{tabular}{lccccc}
\hline $\begin{array}{l}\text { Nivel socioeconómico } \\
\text { de la escuela }\end{array}$ & NSE & Clase & Categórica & $\begin{array}{c}\text { Medio bajo } \\
\text { Medio } \\
\text { Medio alto } \\
\text { Alto }\end{array}$ & 0 \\
\hline $\begin{array}{l}\text { Promedio Simce de } \\
\text { matemáticas de la } \\
\text { escuela }\end{array}$ & Simce & Clase & Numérica & - & 1 \\
$\begin{array}{l}\text { Escuela de perfil } \\
\text { tradicional o } \\
\text { alternativo }\end{array}$ & Perfil & Clase & Categórica & $\begin{array}{c}\text { Tradicional } \\
\text { Alternativo }\end{array}$ & 0 \\
\hline
\end{tabular}

Fuente: Elaboración propia.

Para obtener un indicador categórico de los entornos didácticos de los profesores y poder distinguir sus distintas características se realizó un análisis de grupos o clúster con el algoritmo k-means (Fielding, 2006). Este método busca generar grupos que sean distintos entre sí, pero cuyas observaciones sean similares. Para definir el número de grupos o clúster a extraer se consideró el método del codo (Fielding, 2006). Las variables contempladas fueron D1, D2, D3, D4, D5 y D6. A partir de este análisis, se definió la variable "Entornos" que indica, para cada profesor, el clúster al que pertenece. Este clúster se considera como un indicador del entorno didáctico que genera el profesor.

Para estimar el efecto de los entornos didácticos en la CM se consideraron modelos multinivel con dos niveles (estudiante y clase). Se estimaron tres modelos, los que se describen en la Tabla 4. El primer modelo corresponde al modelo nulo y no incluye ninguna variable independiente, solo efectos aleatorios a nivel del estudiante y de la escuela. Esto permite estimar cómo se distribuye la varianza entre los dos niveles. El segundo modelo incluye los entornos didácticos ("Entornos") como variable independiente, lo que permite estimar su efecto sin controlar por ninguna variable. Finalmente, el tercer modelo incluye las variables de control consideradas en el estudio: "Sexo", "NSE", "Perfil" y "Simce".

Respecto del ajuste de los modelos, se contemplaron varios indices de ajuste (deviance, AIC y BIC), cuya utilidad es que permiten comparar el ajuste de los modelos, así como también su parsimonia. En todos ellos, se cumple que un menor valor indica un mejor ajuste. 
En el caso de la deviance, se pueden hacer test estadísticos que indiquen si su disminución es significativa (Pinheiro, Bates, DebRoy, Sarkar \& Team, 2017).

Tabla 4

Descripción de los modelos multinivel estimados

\begin{tabular}{ccl}
\hline Modelo & Formulación & Descripción \\
\hline 1 & $y_{i j}=\beta_{o}+\mu_{j}+\varepsilon_{i j}$ & Modelo nulo \\
2 & $y_{i j}=\beta_{o}+\beta_{1}$ entornos $_{j}+\mu_{j}+\varepsilon_{i j}$ & Modelo con el efecto de entornos \\
& & en la creatividad matemática. \\
3 & $y_{i j}=\beta_{o}+\beta_{1}$ entornos $_{j}+\beta_{2}$ sexo $_{i j}$ & Modelo con el efecto de entornos \\
& $+\beta_{3}$ NSE $_{j}+\beta_{4}$ perfil $_{j}$ & y variables de control en la \\
& $+\beta_{5}$ simce $_{j}+\mu_{j}+\varepsilon_{i j}$ & creatividad matemática.
\end{tabular}

Fuente: Elaboración propia.

Todos los análisis estadísticos se realizaron con el software R, la definición de los clúster se realizó con la función k-means del paquete Stats (R Core Team, 2016). La estimación de los modelos multinivel se realizó con la función lme del paquete Nlme (Pinheiro et al., 2017).

\section{Resultados}

Los estadísticos descriptivos de los puntajes obtenidos en el instrumento elaborado para evaluar CM se muestran en las Tablas 5, 6 y 7. Los estudiantes pertenecientes a niveles socioeconómicos más altos obtuvieron puntajes mayores, tal como muestra la Tabla 6 , siendo la media del puntaje de los niños del nivel socioeconómico alto más de 5 puntos mayor a la media de los estudiantes pertenecientes a un nivel socioeconómico medio-bajo. El promedio del puntaje de las niñas en la muestra total fue 1,5 puntos mayor al promedio del puntaje de los niños (Tabla 7).

Tabla 5

Estadísticos descriptivos de los puntajes del test de creatividad matemática

\begin{tabular}{ccccc}
\hline $\mathrm{N}$ & Media & $\begin{array}{c}\text { Desviación } \\
\text { estándar }\end{array}$ & Mínimo & Máximo \\
\hline 576 & 13,12 & 6,10 & 0 & 41 \\
\hline
\end{tabular}

Fuente: Elaboración propia. 
338 PENSAMIENTO MATEMÁTICO CREATIVO EN AULAS DE ENSEÑANZA PRIMARIA: ENTORNOS DIDÁCTICOS QUE POSIBILITAN SU DESARROLLO - P. Araya, V. Giaconi, M.V. Martínez

Tabla 6

Estadísticos descriptivos de los puntajes del test de creatividad matemática para los distintos niveles socioeconómicos

\begin{tabular}{lccccc}
\hline NSE & $\mathrm{N}$ & Media & $\begin{array}{c}\text { Desviación } \\
\text { estándar }\end{array}$ & Mínimo & Máximo \\
\hline Medio bajo & 30 & 9,57 & 5,96 & 2 & 28 \\
\hline Medio & 270 & 11,76 & 5,32 & 0 & 31 \\
\hline Medio alto & 63 & 12,94 & 4,46 & 4 & 30 \\
\hline Alto & 213 & 15,39 & 6,69 & 2 & 41 \\
\hline
\end{tabular}

Fuente: Elaboración propia.

Tabla 7

Estadísticos descriptivos de los puntajes del test de creatividad matemática para cada valor de sexo

\begin{tabular}{lccccc}
\hline Sexo & $\mathrm{N}$ & Media & $\begin{array}{c}\text { Desviación } \\
\text { estándar }\end{array}$ & Mínimo & Máximo \\
\hline Mujer & 267 & 14,06 & 6,37 & 0 & 41 \\
\hline Hombre & 309 & 12,30 & 5,74 & 2 & 31 \\
\hline
\end{tabular}

Fuente: Elaboración propia.

Los promedios de los puntajes obtenidos para cada una de las seis dimensiones de entornos didácticos se muestran en la Tabla 8. La dimensión que obtuvo un mayor promedio correspondió a las posibilidades de comunicación entre estudiantes, lo que puede indicar una inclinación de estos profesores a realizar clases con mayor interacción social, al menos en las dos clases filmadas. La dimensión 5 muestra el menor puntaje promedio, lo que indica a modo general una tendencia a no modificar la forma en que se organiza la sala de clases. La dificultad de las tareas propuestas tiene un puntaje promedio de 0,59, mostrando una fuerte variación entre los profesores. En la dimensión 2, se observó una inclinación a presentar los contenidos después de preguntas triviales, siendo poco frecuentes las clases donde las nociones matemáticas eran elaboradas en conjunto con los estudiantes. También se observó variedad en la dimensión 3, donde los profesores estuvieron en un rango de 0,38 , puntaje que indica una forma más pasiva de responder a las producciones matemáticas de los alumnos, hasta un puntaje máximo de 0,75 , lo que indica una forma de respuesta más activa, solicitando justificaciones o devolviendo las preguntas a los estudiantes. 
Tabla 8

Estadísticos descriptivos de los entornos didácticos

\begin{tabular}{llllll}
\hline Variable & $\mathrm{N}$ & Media & $\begin{array}{l}\text { Desviación } \\
\text { estándar }\end{array}$ & Mínimo & Máximo \\
\hline D1 & 21 & 0,59 & 0,11 & 0,44 & 0,92 \\
\hline D2 & 21 & 0,53 & 0,07 & 0,45 & 0,75 \\
\hline D3 & 21 & 0,58 & 0,10 & 0,38 & 0,75 \\
\hline D4 & 21 & 0,68 & 0,09 & 0,47 & 0,81 \\
\hline D5 & 21 & 0,42 & 0,19 & 0,33 & 1,00 \\
\hline D6 & 21 & 0,65 & 0,06 & 0,55 & 0,78 \\
\hline
\end{tabular}

Fuente: Elaboración propia.

Al realizar la conformación de grupos o clúster de acuerdo con las características en las variables D1, D2, D3, D4, D5 y D6, en una primera agrupación, los grupos solo diferían en sus promedios en la variable D5. Esto se explica porque D5 no tiene una relación fuerte con las otras variables y su varianza es mayor en comparación con las otras. Por otro lado, conceptualmente esta variable no distingue aspectos didácticos, como el resto, si no aspectos de organización de la clase. En función del comportamiento estadístico y su definición conceptual, se decidió eliminar a la variable D5 de este análisis. Al rehacer el análisis de grupo con las variables D1, D2, D3, D4 y D6, el método del codo sugería extraer dos o tres grupos. Se extrajeron varias veces dos o tres grupos: al extraer dos grupos los resultados eran estables, es decir, el algoritmo llegaba aproximadamente a los mismos grupos cada vez. Sin embargo, al extraer tres grupos, sus características y tamaño cambiaban, por lo que se extrajeron solamente dos grupos.

En el primer grupo, denominado Entorno A, se ubicaron 10 profesores. En el segundo grupo, denominado Entorno B, se ubicaron 11 profesores. Las características promedio de los profesores en cada grupo se presentan en la Figura 1.

Las aulas del Entorno A mostraron un promedio menor que las del Entorno B en todas las variables, tal como muestra la Figura 1. A modo general, las aulas del Entorno B agrupan a aquellas que tuvieron altos puntajes en todas o en la mayoría de las dimensiones consideradas. 


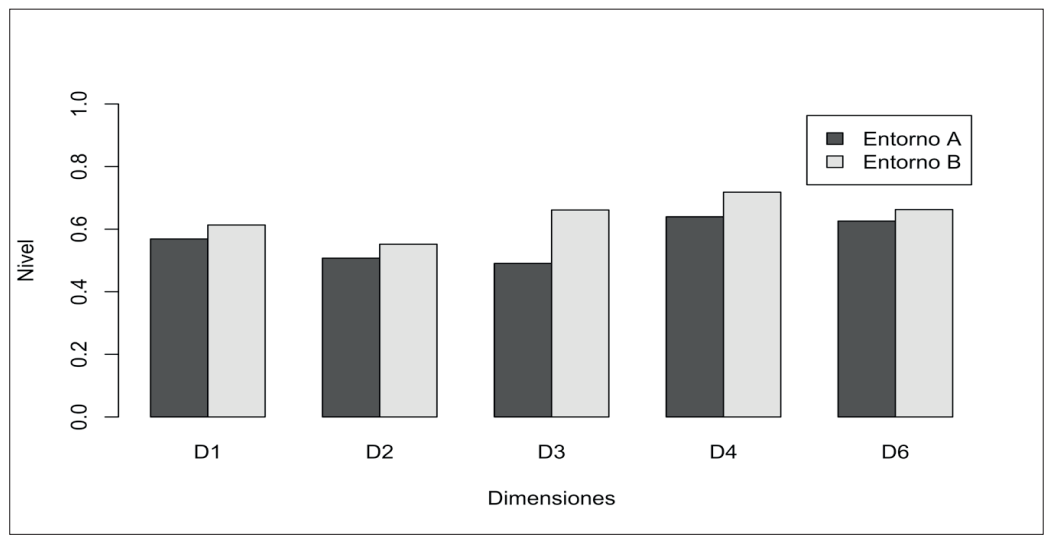

Figura 1. Promedio de cada dimensión en cada grupo.

Fuente: Elaboración propia.

Para ver cómo se distribuían los entornos didácticos de acuerdo con el nivel socioeconómico y el perfil de la escuela se estimaron tablas de contingencia (Tabla 9 y Tabla 10). La Tabla 9 muestra que ambos entornos didácticos se observaron en todos los niveles socioeconómicos, salvo para el nivel medio bajo, en donde solo se encontró el Entorno B. La Tabla 10 muestra que ambos entornos didácticos se observaron en las distintas escuelas. Lo anterior, implica que para esta muestra los entornos A y B no se agruparon en un mismo nivel socioeconómico ni en un mismo perfil de escuela. Este resultado no es generalizable a las escuelas chilenas, ya que la muestra del estudio incluyó a profesores que estuvieron interesados en participar en un taller docente acerca de creatividad y matemáticas, lo que pudo inclinar la muestra hacia profesores particularmente motivados. Sin embargo, esta distribución resultará muy importante para el objetivo general de esta investigación, ya que al contar con distintos entornos en varios NSE y perfiles se podrá identificar el efecto de tales entornos en la CM. 
Tabla 9

Tabla de contingencia entre el nivel socioeconómico de la escuela y el entorno didáctico

\begin{tabular}{|c|c|c|c|}
\hline & & \multicolumn{2}{|c|}{ Entorno didáctico } \\
\hline & & Entorno A & Entorno B \\
\hline \multirow[t]{4}{*}{ Nivel socioeconómico } & Medio bajo & 0 & 1 \\
\hline & Medio & 3 & 6 \\
\hline & Medio alto & 1 & 1 \\
\hline & Alto & 6 & 3 \\
\hline
\end{tabular}

Fuente: Elaboración propia.

Tabla 10

Tabla de contingencia entre el perfil de la escuela y el entorno didáctico

Entorno didáctico

\begin{tabular}{llcc}
\cline { 2 - 4 } & & Entorno A & Entorno B \\
\hline \multirow{2}{*}{ Perfil de la escuela } & Tradicional & 8 & 10 \\
\cline { 2 - 4 } & Alternativo & 2 & 1 \\
\hline
\end{tabular}

Fuente: Elaboración propia.

Para establecer la relación entre la CM de los estudiantes y los entornos didácticos en los que están inmersos, se estimaron tres modelos multinivel (Tabla 11).

El primer modelo (nulo) muestra que un 16\% de la varianza en la CM se explica a nivel aula y un $84 \%$ se explica a nivel individual. Esto da cuenta de que hay una influencia del aula en la creatividad y que esta no depende solamente del individuo. Esto, además, justifica el uso de los modelos multinivel.

En el segundo modelo, se estima el efecto de los entornos didácticos sin incluir ninguna otra variable. El modelo muestra que estar inmerso en el Entorno B tiene un efecto no significativo. Sin embargo, en este modelo probablemente el efecto del entorno didáctico está sesgado, ya que no es controlado por ninguna otra variable. La deviance y el AIC indican un mejor ajuste del modelo 2, pero las diferencias son muy pequeñas. El BIC indica un mejor ajuste del modelo 1.

Finalmente, el modelo 3 indica el efecto del entorno didáctico considerando las variables "NSE", "Simce", "Género" y "Perfil de la 

ENTORNOS DIDÁCTICOS QUE POSIBILITAN SU DESARROLLO - P. Araya, V. Giaconi, M.V. Martínez

escuela". Al tomar en cuenta todos estos criterios, el Entorno B muestra un efecto positivo y significativo sobre la CM. Respecto de las otras variables, el que un estudiante sea de sexo femenino tiene un efecto positivo y significativo. Se observan, además, efectos significativos respecto del grupo socioeconómico, para esta estimación se considera como referencia el nivel socioeconómico medio bajo. El efecto del NSE muestra una diferencia significativa de 5,51 puntos al pertenecer a un NSE alto respecto del NSE medio-bajo. Finalmente, el efecto del puntaje Simce en matemática de la escuela, obtenido por los $4^{\circ}$ años básicos del año 2017, no fue significativo, sin embargo, estuvo asociado a un p-valor de 0,065, lo que sugiere que es importante. Respecto de los índices de ajuste, la deviance y el AIC indican que el modelo 3 tiene un mejor ajuste que el modelo 2, justificando la inclusión de las variables de control.

Tabla 11

Resultados de los modelos multinivel

\begin{tabular}{lccc}
\hline & Modelo 1 & Modelo 2 & Modelo 3 \\
\hline Efectos fijos & & & \\
Intercepto & $13,17^{* *}$ & $12,76^{* *}$ & $-2,60$ \\
Entornos (Entorno B) & & 0,77 & $1,91^{*}$ \\
Sexo (mujer) & & $1,40^{* *}$ \\
NSE (Medio) & & 2,21 \\
NSE (Medio alto) & & $3,42^{\mathrm{a}}$ \\
NSE (Alto) & & $5,51^{* *}$ \\
Perfil (Alternativo) & & $-0,49$ \\
Simce & & & $0,04^{\mathrm{a}}$ \\
\hline Efectos aleatorios & & \\
Varianza entre clases & 6,17 & 6,43 & 0,85 \\
Varianza entre estudiantes & 31,80 & 31,78 & 31,50 \\
\hline Índices de ajuste & & & \\
Deviance & & $3.623,892$ \\
AIC & $3.663,312$ & $3.660,698$ & $3.643,892$ \\
BIC & $3.669,311$ & $3.668,698$ & $3.687,313$ \\
\hline
\end{tabular}

Nota: El superíndice a indica un $p$-valor $<0,1$, el superíndice * indica $p$-valor $<0,05$ y el superíndice ${ }^{* *}$ indica un $p$-valor $<0,01$.

Fuente: Elaboración propia. 
A modo general, se observa que las características del aula de matemática en las que ha indagado esta investigación han resultado relevantes para establecer el nivel de CM evaluado en los alumnos participantes. Esta importancia se refleja en que los estudiantes pertenecientes a las aulas que conformaron el clúster denominado Entornos B, obtuvieron una media de 1,9 puntos mayor a la de los estudiantes cuyos profesores estuvieron en el clúster denominado Entornos A, una vez que fueron incorporadas las variables de control "Género", "NSE", "Simce" y "Perfil de la escuela".

\section{Discusión}

Como hemos mencionado anteriormente, la CM se relaciona con la capacidad de crear ideas matemáticas que son novedosas desde la perspectiva de quien las genera. En la actualidad, la literatura internacional ha propuesto formas de evaluar esta habilidad, generalmente mediante el uso de problemas de respuesta múltiple, que permiten a los sujetos generar distintas soluciones a una misma pregunta. Dichas soluciones poseen distintos niveles de sofisticación y pueden analizarse a partir de las dimensiones fluidez, flexibilidad y originalidad. La presente investigación evaluó de manera confiable la CM de 576 estudiantes pertenecientes a 21 cursos y 17 escuelas de la Región Metropolitana.

Sobre la evaluación de la creatividad matemática (objetivo 1), la prueba de problemas de solución múltiple aplicada a los estudiantes permitió identificar desempeño en las tres dimensiones de CM reportadas por la literatura: fluidez, flexibilidad y originalidad. Los resultados de la prueba indican que, en promedio, los estudiantes pueden desarrollar múltiples soluciones, diversas estrategias y encontrar soluciones originales. Además, los resultados muestran que los puntajes tienen mucha variación, considerando la desviación estándar y el rango (distancia entre máximo y mínimo). Esta amplia variación se observa en todos los niveles socioeconómicos y en ambos sexos. Esto da muestra de que, independientemente del NSE y sexo, hubo estudiantes que lograron proponer solo respuestas triviales a los problemas y otros, en cambio, generaron gran cantidad de respuestas con altos niveles de sofisticación. 

ENTORNOS DIDÁCTICOS QUE POSIBILITAN SU DESARROLLO - P. Araya, V. Giaconi, M.V. Martínez

Las dimensiones del aula matemática consideradas en este estudio permitieron caracterizar los entornos didácticos en dos grupos (objetivo 2). Las aulas del grupo B, en comparación con el grupo A, se caracterizaron por un estilo de enseñanza más activa. Las tareas que presentaron los profesores durante la clase fueron más desafiantes, empleando por lo general problemas de alta demanda cognitiva. La presentación de los contenidos fue ligeramente más participativa que la del grupo A, requiriendo que los estudiantes se enfrentaran a preguntas desafiantes antes de la presentación de las nociones e ideas matemáticas trabajadas en la clase. Un aspecto que distinguió claramente ambos grupos fue la manera en que reaccionaron a las preguntas y contribuciones de los estudiantes: mientras que el grupo A tendió a dar las respuestas a las preguntas, el grupo B usó con mayor frecuencia estrategias como devolver la pregunta a la clase, o pedir que un estudiante justificara sus soluciones.

Se observó que, en la mayoría de las aulas participantes, los profesores gestionaron la comunicación en el aula de forma abierta e integrada, propiciando que los estudiantes se comunicaran en grupos, parejas, con el profesor y con toda la clase. Puede que esta característica haya estado presente en muchas de las aulas del estudio puesto que los profesores participaron del estudio de manera voluntaria, ya que estaban interesados en conocer más acerca de la creatividad y el aula. Nuevamente, esta característica fue mayor en las aulas del grupo B.

Se observa que la capacidad de los profesores para variar la dificultad de los problemas también resultó ser mayor en los cursos del grupo B. Los profesores del grupo B mostraron mayor conocimiento sobre distintas formas de hacer más difícil un problema para sus estudiantes, por ejemplo, variaciones en el orden sintagmático y operativo, inclusión de datos irrelevantes, entre otras.

Respecto de cómo los entornos didácticos se relacionan con la CM de los estudiantes (objetivo 3), los resultados de este estudio confirman el efecto del aula sobre la CM de los estudiantes, mediante el uso de modelos multinivel. Esto reafirma la relevancia de indagar en profundidad en las características de la enseñanza que permiten 
a los estudiantes un mayor desarrollo de esta habilidad. Lo anterior coincide con lo encontrado en las investigaciones de Kanhai y Singh (2016), Kwon, Park y Park (2006), Sarrazy y Novotná, (2013) y Tabach y Friedlanyer (2013) quienes mostraron la influencia del aula en la CM a partir de distintas investigaciones realizadas en contextos escolares de Europa y Norteamérica.

Los modelos multinivel estimados muestran un efecto positivo y significativo de las aulas del grupo B, el cual puede identificarse una vez que se consideran factores (variables de control) que pueden incidir en la CM como son "NSE", "Género", "Perfil de la escuela" y "Simce". Las dimensiones que constituyen los entornos didácticos analizados en este estudio concuerdan con otras variables que han mostrado influencia sobre la CM en otras investigaciones. Por ejemplo, en las aulas del grupo B los estudiantes tuvieron más posibilidades de comunicación. Los resultados de Hershkowitz, Tabach y Dreyfus (2017) explican este hallazgo al analizar el modo en que las ideas creativas pueden tener cabida en la sala de clases, ya que a menudo una contribución nueva puede "ver la luz" en el aula a través de la acción comunicativa entre pares, que permite la construcción colectiva de una idea. Dicha comunicación es más potente si el profesor promueve las discusiones respecto de ideas matemáticas. Otra dimensión considerada guardó relación con la capacidad de los profesores de variar la dificultad de los problemas matemáticos. Sarrazy (2002) explica la influencia de esta variable sobre el pensamiento matemático creativo señalando que una alta capacidad para generar problemas matemáticos que varíen en distintos criterios de dificultad lleva a los estudiantes a un compromiso cognitivo mayor, pues no pueden aplicar algoritmos ya conocidos sin detenerse en su pertinencia para dar solución al problema dado.

Al revisar los factores que pueden incidir en el fenómeno estudiado ("NSE", "Género del estudiante", "Perfil de la escuela" y "Simce") se observa que el nivel socioeconómico al que pertenecen los alumnos tiene un efecto significativo en su nivel de CM. Esto coincide con los resultados de Singh (1990) quien mostró el efecto de la clase social en la CM de estudiantes de la India pertenecientes a 

ENTORNOS DIDÁCTICOS QUE POSIBILITAN SU DESARROLLO - P. Araya, V. Giaconi, M.V. Martínez

distintas castas. Por lo general, las investigaciones realizadas en países desarrollados han dejado fija esta variable para indagar el efecto de otros factores sobre la CM (por ejemplo, Kwon, Park \& Park, 2006; Mann, 2009). En modelos educativos altamente segregados como el chileno, resulta necesario incorporar de manera controlada la variable socioeconómica, ya que la mayoría de las mediciones que se realizan en educación están permeadas por los contextos socioculturales de los sujetos participantes.

Los hallazgos de este estudio muestran diferencias de género sobre la CM, encontrándose un efecto significativo que favorece a las mujeres. Este resultado coincide con estudios norteamericanos (Evans, 1964; Jensen, 1973; Mann, 2009; Prousse, 1967). El estudio de Mann (2009) mediante modelos de regresión analizó el efecto del género sobre la $\mathrm{CM}$ encontrando que este factor contribuye a explicar un $0,47 \%$ de varianza y que la diferencia es favorable hacia las mujeres. Evans (1964) analizó los datos de estudiantes entre octavo y quinto grado. Las niñas de octavo y séptimo grado superaron a los niños con diferencias significativas. No se encontraron diferencias significativas de género para los grados restantes. Jensen (1973) investigó el desempeño en $\mathrm{CM}$ en estudiantes de sexto grado en tres escuelas: si bien la diferencia en las puntuaciones obtenidas entre los establecimientos no fue significativa, sí se observaron diferencias significativas a favor de las mujeres en una de las tres escuelas y ninguna diferencia en las otras dos. Prouse (1967) investigó la creatividad en matemática en estudiantes de séptimo grado e informó una diferencia de medias significativa en las puntuaciones compuestas de creatividad que favorecen a las mujeres. Baer y Kauffman (2008) revisaron la literatura sobre creatividad general, encontrando que la mayoría de las investigaciones basadas en pruebas de creatividad evidenciaron una diferencia favorable hacia las mujeres y niñas. Estos hallazgos contrastan con la evidencia robusta acerca del menor rendimiento matemático de las mujeres obtenido en evaluaciones estandarizadas. Por ejemplo, en los resultados en matemática de la prueba PISA (2012), los niños superan a las niñas por 11 puntos como promedio de los países de la OCDE. En los países latinoamericanos esa brecha es aún peor. Por ejemplo, según Mizala (2014), solo un tercio de los estudiantes chilenos con altos rendimientos en matemática en PISA 2009 fueron mujeres. Es pertinente citar a 
Mann (2009) quien propone que es posible que mediante la evaluación de la CM se esté reconociendo en las niñas un talento poco estudiado, ya que esta habilidad corresponde a uno de los tipos de pensamiento más sofisticados dentro de la matemática (Lithner, 2008). Como sea, estos resultados abren una ventana para indagar más profundamente en este tema.

Sobre el efecto de pertenecer a una escuela alternativa la muestra de este estudio involucró algunas escuelas artísticas, Montessori o Freinet, las que en sus proyectos educativos mostraban como un objetivo explícito el desarrollo de la creatividad en sus estudiantes. Los resultados de esta investigación no mostraron ningún efecto significativo sobre la creatividad matemática de los estudiantes, sin embargo, la muestra solo contenía tres escuelas de este tipo, por lo que los resultados no fueron concluyentes en este tema. Si consideramos que una de las razones de los padres para que sus hijos ingresen en estas escuelas es que puedan desarrollar su creatividad, es evidente la importancia de este aspecto y sería interesante contar con mayores estudios que indaguen esta relación.

Con respecto al promedio de matemática en la prueba Simce de $4^{\circ}$ año básico 2017, el efecto resultó no ser significativo considerando el nivel de significancia usual de $5 \%$, pero sí lo fue si se considera un nivel del 10\%. Podemos inferir que la evaluación de conocimientos matemáticos curriculares que realiza Simce y la evaluación de la creatividad matemática realizada en este estudio difieren sustancialmente, ya que si evaluaran constructos equivalentes el efecto habría sido aún mayor. Sin embargo, este dato debe ser tomado con distancia, ya que el Simce contemplado en este estudio es un promedio obtenido por todos los alumnos que el año anterior estuvieron en cuarto básico en cada colegio. Un futuro estudio que explore la relación entre la creatividad matemática y los resultados individuales del Simce permitiría ahondar en las diferencias que se obtienen en ambas evaluaciones.

El objetivo 4 se propuso dar cuenta de características de los entornos didácticos que pueden favorecer o inhibir la CM de los estudiantes. Este estudio logró identificar que los entornos didácticos 

ENTORNOS DIDÁCTICOS QUE POSIBILITAN SU DESARROLLO - P. Araya, V. Giaconi, M.V. Martínez

presentes en las aulas del grupo B favorecieron la CM de los estudiantes. Las características de este grupo fueron descritas anteriormente. Sin embargo, una limitación de este estudio tiene que ver con la dificultad de aislar las dimensiones de los entornos didácticos que impactan en mayor y menor medida la CM de los estudiantes, debido a que el número de clases en la muestra imposibilita agregar más variables a nivel clase en el modelo multinivel. Esta es una posible línea de trabajo para continuar con este estudio, así como también lo es enriquecer el análisis al complementarlo con un enfoque cualitativo.

En síntesis, los hallazgos de este estudio confirman la influencia del aula escolar en el desarrollo de la capacidad inventiva en ámbitos matemáticos, y contribuyen a identificar algunas características del aula y del profesorado que, en conjunto, tienen un efecto positivo en su desarrollo, a saber: el involucramiento activo de los estudiantes en el trabajo con las ideas matemáticas de la clase, la comunicación entre distintos actores del aula, la capacidad del maestro de retroalimentar las ideas o preguntas de estudiantes dando mayor responsabilidad al alumno sobre la construcción de ideas matemáticas, el trabajo en tareas que resulten desafiantes, y la capacidad del maestro de variar la dificultad de los problemas que da a sus alumnos. Estas características, en su conjunto, logran impactar positivamente la habilidad estudiada una vez que se controlan factores como NSE, género y Simce. Estos resultados muestran la importancia de que los maestros involucren a los estudiantes en la actividad matemática de la clase con actividades que los desafíen y les permitan pensar en cuestiones no triviales, brindando el espacio para que estos realicen aportes y contribuyan en la búsqueda de soluciones matemáticas.

Un estilo de enseñanza que estimula a los alumnos a generar ideas, preguntas y estrategias matemáticas tiene incidencias no solo en los resultados académicos, sino que también en su capacidad de pensar creativamente.

Este estudio no llega a adentrarse en las causas que explican estilos pedagógicos activos en la enseñanza de la matemática, cuestión que resultaría importante de mirar más profundamente en estudios futuros, así como elementos que permitan desarrollar en el 
profesorado las competencias que los capaciten para potenciar en sus estudiantes pensamiento creativo en el ámbito matemático.

\section{Referencias}

Baer, J. \& Kaufman, J. (2008). Gender differences in creativity. The Journal of Creative Behavior, 42(2), 75-105. https://doi. org/10.1002/j.2162-6057.2008.tb01289.x

Barquero, B., Richter, A., Barajas, M., y Font, V. (2014). Promoviendo la creatividad matemática a través del diseño colaborativo de c-unidades. En M. T. González, M. Codes, D. Arnau y T. Ortega (Eds.), Investigación en educación matemática (pp. 157-166). Salamanca: Sociedad Española de Investigación en Educación Matemática.

Bickel, R. (2007). Multilevel analysis for applied research: It’s just regression! New York: Guilford Press.

Brousseau, G. (2007). Iniciación al estudio de la teoría de las situaciones didácticas. Buenos Aires: Libros del Zorzal.

Brousseau, G., Sarrazy, B., \& Novotná, J. (2014). Didactic contract in mathematics education. En S. Lerman (Ed.), Encyclopedia of mathematics education (pp. 153-159). Dordrecht: Springer Netherlands.

Csikszentmihalyi, M. (2000). Implications of a systems perspective for the study of creativity. In R. J. Sternberg (Ed.), Handbook of creativity (pp. 313-335). Cambridge: Cambridge University Press.

Dreyfus, T. \& Eisenberg, T. (1996). On different facets of mathematical thinking. En R. J. Sternberg \& T. Ben-Zeev (Eds.), The nature of mathematical thinking (pp. 253-284). Mahwah: Lawrence Erlbaum.

European Commission, EC. (2006). Recommendation 2006/962/EC of the European Parliament and of the Council of 18 December 2006 on key competences for lifelong learning, Official Journal L 394 of 30.12.2006, 10-18.

Ervynck, G. (1991). Mathematical creativity. En A. M. Thinking \& D. Tall (Eds.), Advanced mathematical thinking (pp. 42-53). Dordrecht: Springer.

Evans, E. W. (1964). Measuring the ability of students to respond in creative mathematical situations at the late elementary and early junior high school level. Dissertation Abstracts International, 25(12), 7-107.

Fielding, A. (2006). Cluster and classification techniques for the biosciences. New York: Cambridge University Press. 
350 PENSAMIENTO MATEMÁTICO CREATIVO EN AULAS DE ENSEÑANZA PRIMARIA: ENTORNOS DIDÁCTICOS QUE POSIBILITAN SU DESARROLLO - P. Araya, V. Giaconi, M.V. Martínez

Ginsburg, H. (1996). Toby's math. En R. J. Sternberg \& T. Ben-Zeev (Eds.), The nature of mathematical thinking (pp. 175-282). Mahwah: Lawrence Erlbaum.

Hannula, M. (2014). Affect in mathematics education. En S. Lerman (Ed.), Encyclopedia of mathematics education (pp. 23-27). Dordrecht: Springer Netherlands.

Hannula, M., Di Martino, P., Pantziara, M., Zhang, Q., Morselli, F., HeydMetzuyanim, E. ... \& Goldin, G. (2016). Attitudes, beliefs, motivation, and identity in mathematics education. En M. Hannula, P. Di Martino, M. Pantziara, Q. Zhang, F. Morselli, E. Heyd-Metzuyanim, E. ... \& G. Goldin (Eds.), Attitudes, beliefs, motivation and identity in mathematics education (pp. 1-35). New York: Springer International Publishing.

Hershkowitz, R., Tabach, M., \& Dreyfus, T. (2017). Creative reasoning and shifts of knowledge in the mathematics classroom. ZDM Mathematics Education, 49(1), 25-36. https://doi.org/10.1007/s11858-016-0816-6

Jensen, L. (1973). The relationships among mathematical creativity, numerical aptitude and mathematical achievement. Dissertation Abstracts International, 34(5), 21-68.

Joët, G., Usher, E., \& Bressoux, P. (2011). Sources of self-efficacy: An investigation of elementary school students in France. Journal of Educational Psychology, 103(3), 6-49. https://doi.org/10.1037/ a0024048

Kanhai, A. \& Singh, B. (2016). Some environmental and attitudinal characteristics as predictors of mathematical creativity. International Journal of Mathematical Education in Science and Technology, 48(3), 327337. https://doi.org/10.1080/0020739x.2016.1241435

Kattou, M., Kontoyianni, K., Pitta-Pantazi, D., \& Christou, C. (2013). Connecting mathematical creativity to mathematical ability. ZDM Mathematics Education, 45(2), 167-181. https://doi.org/10.1007/ s11858-012-0467-1

Kwon, O. N., Park, J. H., \& Park, J. S. (2006). Cultivating divergent thinking in mathematics through an open-ended approach. Asia Pacific Education Review, 7(1), 51-61. https://doi.org/10.1007/bf03036784

Lee, K., Hwang, D., \& Seo, J. (2003). A development of the test for mathematical creative problem solving ability. Journal of the Korea Society of Mathematical Education, 7(3), 163-189.

Leikin, R. (2013). Evaluating mathematical creativity: The interplay between multiplicity and insight. Psychological Test and Assessment Modeling, 55(4), 385-400. 
Leikin, R., \& Pitta-Pantazi, D. (2013). Creativity and mathematics education: The state of the art. ZDM Mathematics Education, 45(2), 159-166. https://doi.org/10.1007/s11858-012-0459-1

Lev-Zamir, H. \& Leikin, R. (2013). Saying versus doing: teachers' conceptions of creativity in elementary mathematics teaching. ZDM Mathematics Education, 45(2), 295-308. https://doi.org/10.1007/s11858-012-0464-4

Liljedahl, P. (2013). Illumination: An affective experience? The International Journal on Mathematics Education, 45(2), 253-265. https://doi. org/10.1007/s11858-012-0473-3

Liljedahl, P. (2016). Building thinking classrooms: Conditions for problemsolving. En P. Felmer, E. Pehkonen, \& J. Kilpatrick (Eds.), Posing and solving mathematical problems (pp. 361-386). New York: Springer International Publishing.

Liljedahl, P. \& Sriraman, B. (2006). Musing on mathematical creativity. For the Learning of Mathematics, 26(1), 20-23.

Linnenbrink, E. A. \& Pintrich, P. R. (2004). Role of affect in cognitive processing in academic contexts. En D. Y. Dai \& R. J. Sternberg (Eds.), Motivation, emotion, and cognition; integrative perspectives on intellectual functioning and development (pp. 57-88). Mahwah: Lawrence Erlbaum.

Lithner, J. (2008). A research framework for creative and imitative reasoning. Educational Studies in Mathematics, 67(3), 255-276. https://doi. org/10.1007/s10649-007-9104-2

Mann, E. (2006). Creativity: The essence of mathematics. Journal for the Education of the Gifted, 30(1), 236-230. https://doi.org/10.4219/jeg2006-264

Mann, E. (2009). The search for mathematical creativity: Identifying creative potential in middle school students. Creativity Research Journal, 21(4), 338-348. https://doi.org/10.1080/10400410903297402

Mann, E., Chamberlin, S., \& Graefe, A. (2017). The prominence of affect in creativity: Expanding the conception of creativity in mathematical problem solving. En R. Leikin, Roza \& B. Sriraman (Eds.), Creativity and Giftedness (pp. 57-73). Cham: Springer.

Ministerio de Educación de Chile, Mineduc. (2012). Bases curriculares 2012: educación básica matemática. Recuperado de http://www. curriculumenlineamineduc.cl/605/articles-34961_Bases.pdf

Mizala, A. (2014). Mujeres, matemáticas, carrera y brecha familiar. Recuperado de http://www.comunidadmujer.cl/2014/01/columnamujeresmatematicas-carreras-y-brecha-salarial/ 
352 PENSAMIENTO MATEMÁTICO CREATIVO EN AULAS DE ENSEÑANZA PRIMARIA: ENTORNOS DIDÁCTICOS QUE POSIBILITAN SU DESARROLLO - P. Araya, V. Giaconi, M.V. Martínez

Murillo, F. (2008). Los modelos multinivel como herramienta para la investigación educativa. Magis, Revista Internacional de Investigación en Educación, 17(1), 45-62.

Nohda, N. (July, 2000). Teaching by open-approach method in Japanese mathematics classroom. Proceedings of the 24th Conference of the International Group for the Psychology of Mathematics Education PME, Hiroshima, Japan.

Novotná, J. \& Sarrazy, B. (2010). Teacher's didactical variability and its role in mathematics education. CERME 6-Working group 9, 1625.

Novotná, J. \& Sarrazy, B. (2011). Didactical variability in teacher education. En O. Zaslavsky, \& P. Sullivan (Eds.), Constructing knowledge for teaching secondary mathematics (pp. 103-115). Boston: Springer US.

Pehkonen, E. (1997). The state of art in mathematical creativity. ZDM Mathematics Education, 29(3), 63-67. https://doi.org/10.1007/s11858997-0001-z

Piirto, J. (1999). A survey of psychological studies in creativity. En A. Fishkin, B. Cramond, \& P. Olszewski-Kubilius (Eds.), Investigating creativity in youth: research methods (pp. 27-49) Cresskill, NY: Hampton Press.

Pinheiro, J., Bates, D., DebRoy, S., Sarkar, D., \& Team, R. (2017). _nlme: Linear and nonlinear mixed effects models. R package version 3.1-131. Recuperado de https://CRAN.R-project.org/package=nlme

Prouse, H. (1967). Creativity in school mathematics. Mathematics Teacher, 60(3), 876-879.

R Core Team (2016). R: A language and environment for statistical computing. $R$ Foundation for Statistical Computing, Vienna, Austria. Recuperado de https://www.R-project.org/.

Sarrazy, B. (2002). Effects of variability of teaching on responsiveness to the didactic contract in arithmetic problem-solving among pupils of 9-10 years. European Journal of Psychology of Education, 17(4), 321-341. https://doi.org/10.1007/bf03173589

Sarrazy, B. \& Novotná, J. (2005). Didactical contract: Theoretical frame for the analysis of phenomena of teaching mathematics. En J. Novotná (Ed.), Proceedings SEMT 05 (pp. 33-44). Prague: Charles University, Faculty of Education.

Sarrazy, B. \& Novotná, J. (2013). Didactical contract and responsiveness to didactical contract: A theoretical framework for enquiry into students' creativity in mathematics. ZDM Mathematics Education, 45(2), 281-293. https://doi.org/10.1007/s11858-013-0496-4 
Sheffield, L. (2013). Creativity and school mathematics: Some modest observations. ZDM Mathematics Education, 45(2), 325-332. https:// doi.org/10.1007/s11858-013-0484-8

Sheffield, L. (2017). Dangerous myths about "gifted" mathematics students. ZDM Mathematics Education. 49(1), 13-23. https://doi.org/10.1007/ s11858-016-0814-8

Sinclair, N., Freitas, E., \& Ferrara, F. (2013). Virtual encounters: The murky and furtive world of mathematical inventiveness. ZDM Mathematics Education, 45(3), 239-252. https://doi.org/10.1007/s11858-012-0465-3

Singer, F., Sheffield, L., \& Leikin, R. (2017). Advancements in research on creativity and giftedness in mathematics education: Introduction to the special issue. ZDM Mathematics Education, 49(1), 5-12. https:// doi.org/10.1007/s11858-017-0836-x

Singh, B. (1987). The development of tests to measure mathematical creativity. International Journal of Mathematical Education in Science and Technology, 18(2), 181-186. https://doi.org/10.1080/0020739870180203

Singh, B. (1990). Differences in mathematical creativity of middle school children of different social groups. International Journal of Mathematical Education in Science and Technology, 21(4), 541-544. https://doi. org/10.1080/0020739900210404

Smith, M. \& Stein, M. (1998). Selecting and creating mathematical tasks: From research to practice. Mathematics teaching in the middle school, 3(5), 344-50.

Sriraman, B. (2009). The characteristics of mathematical creativity. ZDM Mathematics Education, 41(3), 13-27. https://doi.org/10.1007/s11858008-0114-z

Sternberg, R. (2004). The domain generality versus specificity debate: How should it be posed? En J. C. Kaufman \& J. Baer (Eds.), Creativity across domains: Faces of the muse (pp. 299-306). Hillsdale, New Jersey: Lawrence Erlbaum.

Tabach, M. \& Friedlander, A. (2013). School mathematics and creativity at the elementary and middle-grade levels: how are they related? ZDM Mathematics Education, 45(2), 227-238. https://doi.org/10.1007/ s11858-012-0471-5

Torrance, E. P. (1974). Torrance tests of creative thinking. Bensenville: Scholastic Testing Service.

Vergnaud, G. (1983). A classification of cognitive tasks and operations of thought involved in addition and subtraction problems. En T. P. Carpenter, J. M. Moser, \& T. A. Romberg (Eds.), Addition and 
354 PENSAMIENTO MATEMÁTICO CREATIVO EN AULAS DE ENSEÑANZA PRIMARIA: ENTORNOS DIDÁCTICOS QUE POSIBILITAN SU DESARROLLO - P. Araya, V. Giaconi, M.V. Martínez

subtraction: A cognitive perspective (pp. 39-59). New Jersey: Lawrence Erlbaum Associates.

Voica, C. \& Singer, F. (2013). Problem modification as a tool for detecting cognitive flexibility in school children. ZDM Mathematics Education, 45(2), 267-279. https://doi.org/10.1007/s11858-013-0492-8

Recibido: 05/11/2018

Aceptado: 28/03/2019 
Anexo 1: Ítems que componen test de creatividad matemática

1. A continuación, hay 16 puntos dispuestos a $1 \mathrm{~cm}$ de distancia uno de otro. Forme figuras cuya área sea $2 \mathrm{~cm}^{2}$ uniendo puntos. Dibuje tantas figuras distintas como sea posible.

\section{Ejemplo}

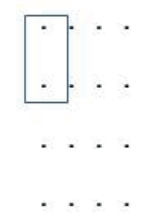

2. Utilizando los números que aparecen en el rectángulo proponga cálculos cuyo resultado final sea 30 . Obtenga tantas respuestas distintas como pueda.

\begin{tabular}{|ccccccc|}
\hline 18 & 2 & 10 & 12 & & \\
& 40 & & 48 & & 3 & 20 \\
& & & & & & \\
& 90 & & 15 & & \\
\hline
\end{tabular}

Por ejemplo: $10+10+10$ y $10+20$

3. Divida un cuadrado y coloree algunas de sus partes, de manera tal que lo coloreado y lo no coloreado tengan la misma área.

Pista: El siguiente ejemplo muestra un círculo. Tú debes aplicar la instrucción a un cuadrado.

(1)

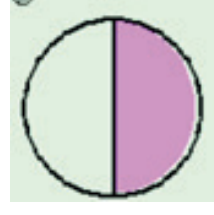

(2)

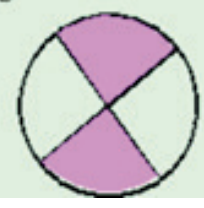

(3)

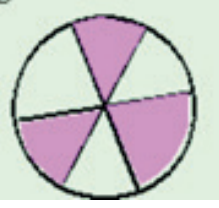


356 PENSAMIENTO MATEMÁTICO CREATIVO EN AULAS DE ENSEÑANZA PRIMARIA: ENTORNOS DIDÁCTICOS QUE POSIBILITAN SU DESARROLLO - P. Araya, V. Giaconi, M.V. Martínez

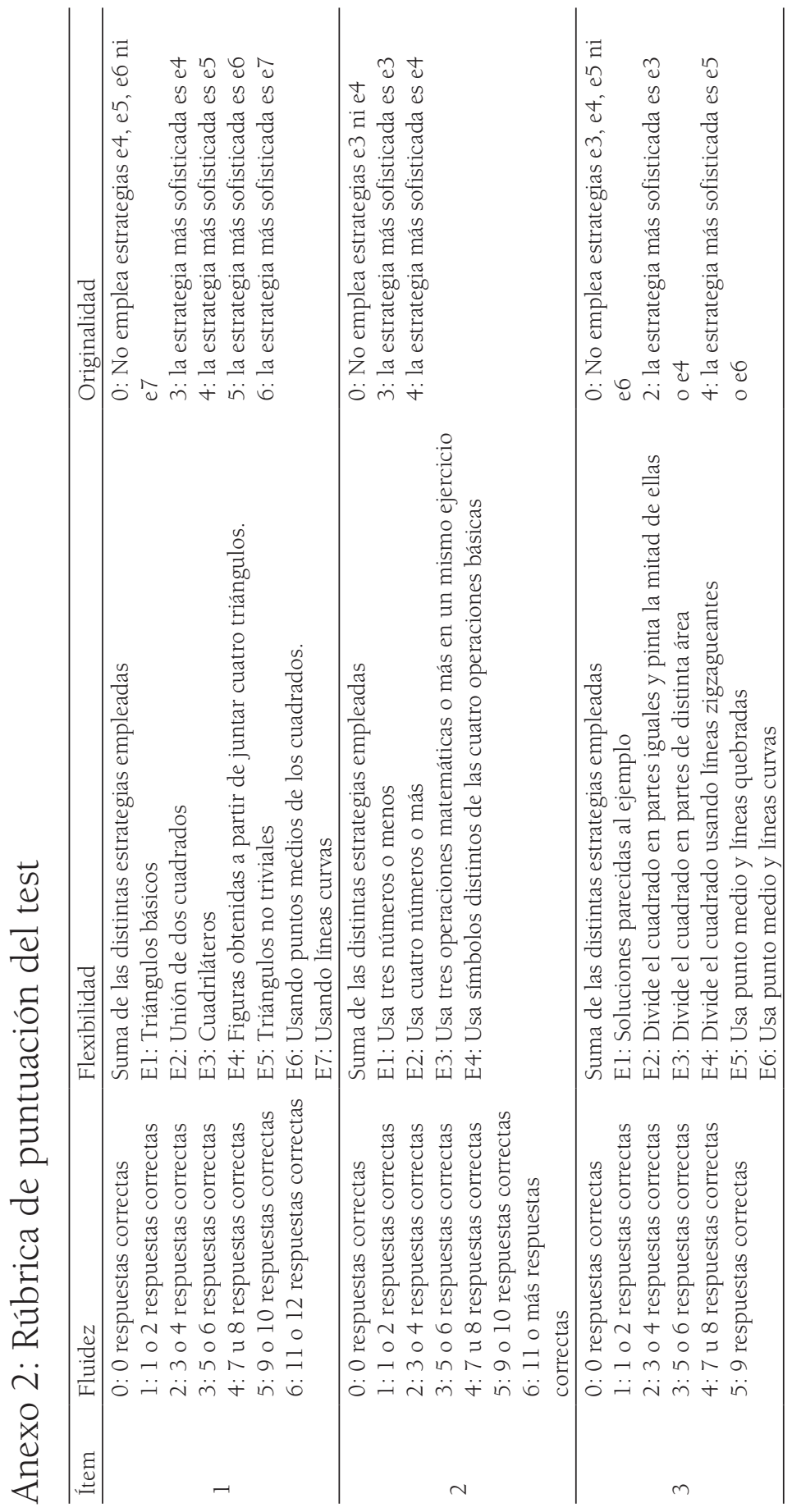

